Krzysztof Oprzędkiewicz*

\title{
A Strejc model-based, semi-fractional (SSF) transfer function model
}

\section{Introduction}

Mathematical models of dynamic systems using fractional-order differentiation have been investigated by many authors (see for example: Podlubny (1999), Busłowicz (2008), Kaczorek (2011)) $[1,4,14,15]$. The fractional-order model in many situations better describes complex and not known exactly plants or processes.

A transfer function is one of most useful models applied to describe elementary properties of control plants and the synthesis of control systems. With the use of typical models, proposed by Strejc or Kupfmuller a huge class of real control plants and processes can be described (see for example Larminat (2007) [6]).

The goal of this paper is to present proposed by author the Strejc-model based transfer function model, which can be useful to describe high-order aperiodic control plants. The model can be easily implemented at MATLAB/SIMULINK platform and their parameters can be identified with the use of the least-square method. In paper the following problems will be presented:

- the proposed SSF model,

- the identification of the proposed SSF model,

- an Example.

\section{The proposed SSF model}

The proposed model of dynamic system consists of two parts: the "classic" Strejc model without delay and the fractional part. The whole model is described with the use of transfer function (1):

$$
G(s)=\frac{k}{\left(T_{M} s+1\right)^{M}\left(T_{\alpha} s^{\alpha}+1\right)}
$$

* AGH University of Science and Technology, Krakow, Poland 
In (1) $T_{M}, M$ and $k$ denote the parameters of "classic" Strejc model: time constant, rank and steady-state gain respectively. $T_{\alpha}$ and $\alpha \in \boldsymbol{R}$ denote parameters of fractional transfer function. In further considerations the model (1) will be called the SSF (Strejc semi fractional) model.

A sense to propose the SSF model described by (1) consists in fact, that during identification of the Strejc model without delay the most hard parameter to estimate is the order of model $M$. The typical situation is, that the both $M$-th order and $M+1-$ th order models are inaccurate and the best accuracy of model seems to be located "between" $M$ and $M+1$. An introduction of the fractional part is expected to increase the accuracy of model, because the fractional order is able to better describe the real plant, which the order is really unknown.

Assume, that the fractional factor sa in (1) is approximated with the use of Oustaloup's Recursive Approximation (ORA) method. It can be expressed as follows (see for example Merrkikh-Bayat (2012) [7]):

$$
s^{\alpha} \cong k_{f} \prod_{n=1}^{N} \frac{1+\frac{s}{\mu_{k}}}{v_{k}}
$$

In (2) $N$ denotes the order of approximation, coefficients $\mu_{k}$ and $v_{k}$ are determined by a frequency range, for which the approximation is required to be built. If the frequency range is defined by border values $\omega_{l}$ and $\omega_{h}$, then coefficients $\mu_{k}$ and $v_{k}$ are expressed as underneath:

$$
\begin{aligned}
& \mu_{1}=\omega_{l} \sqrt{\eta} \\
& v_{n}=\mu_{n} \gamma, \quad n=1, \ldots, N \\
& \mu_{n}=v_{n} \eta, \quad n=1, \ldots, N-1
\end{aligned}
$$

Where:

$$
\begin{aligned}
& \gamma=\left(\frac{\omega_{h}}{\omega_{l}}\right)^{\frac{\alpha}{N}} \\
& \eta=\left(\frac{\omega_{h}}{\omega_{l}}\right)^{\frac{1-\alpha}{N}}
\end{aligned}
$$

The gain $k_{f}$ is adjusted after calculations to assure the convergence the model to real plant in a steady-state.

During practical use the ORA approximation described by (2)-(4) a non-trivial problem is the proper to assign the approximation parameters: the both border frequencies $\omega_{l} \omega_{h}$, and the 
approximation order $N$. Values of these parameters have a significant influence on the correct run of the model built with the use of this approximation. The method of estimation these parameters for fractional $P I \mathrm{a} D^{\mu}$ controller was presented by Merrikh-Bayat (2012) [7], but the method dedicated to models has been not presented. The most simple method to assign these parameters is an application of numerical methods.

The rest of model parameters: the order of Strejc model $M$, the fractional order a and the both time constants: $T_{m} T_{\alpha}$ can be also estimated with the use of least square method and the step response of real plant. This will be shown in the next section.

The SSF model described by (1)-(4) is a function of the number of parameters, which can be divided into the following main functional parts:

- The order of fractional differentiation a,

- The time constants: $T_{m}$ and $T_{\alpha}$,

- The orders of the both parts: $M$ and $N$.

- The frequency range, determined by $\omega_{l}$ and $\omega_{h}$.

Assume, that all of the model parameters build vectors of parameters associated to above functional parts:

$$
\begin{aligned}
& v_{o}=[M ; N] \in V_{o} \subset Z^{2} \\
& v_{\alpha}=[\alpha] \in V_{\alpha} \subset R \\
& v_{T}=\left[T_{M} ; T_{\alpha}\right] \in V_{T} \subset R^{2} \\
& v_{\omega}=\left[\omega_{l} \omega_{h}\right] \in V_{\omega} \subset R^{2}
\end{aligned}
$$

In (5) capital letter " $V$ " denotes a set of all sensible from physical point of view model parameters. All the above vectors build the vector of the SSF model parameters, denoted by $v$ and $V$ respectively:

$$
v=\left[v_{o} ; v_{\alpha} ; v_{T} ; v_{\omega}\right] \in V \subset Z^{2} \times R \times R^{2} \times R^{2}
$$

The step response of the SSF model described by (1)-(6) is a function of vector $v$ and it can be expressed as underneath:

$$
y(v, t)=L^{-1}\left\{\frac{k}{s\left(T_{n} s+1\right)^{n}\left(T_{\alpha} s^{\alpha}+1\right)}\right\}
$$

In (7) the fractional factor sa is expressed by ORA approximation (2)-(4). The function (7) can be calculated with the use of ORA approximation described by (2)-(4) and MATLAB/ SIMULINK. 


\section{The identification of the proposed SSF model}

The measure of the accuracy of the model described above can be a cost function expressed by (8):

$$
I(v)=\int_{0}^{\infty}\left(y(v, t)-y_{e}(t)\right)^{2} d t
$$

In (8) $y(v, t)$ denotes the step response of the SSF model, described by (7), $y_{e}(t)$ denotes an experimental step response of a real plant. To assure a convergence of integral (8) the steadystate of step response of model expressed by (7) must go to the steady-state of step response real plant $y(t)$. This can be easily met by the properly assigned value of steady-state gain $k$.

The search for a SSF model (1) well describing a plant described by an experimental step response consists in finding such a vector $v_{\min } \in V$, for which the cost function (8) will be minimal. It can be expressed as underneath:

$$
I_{\min }\left(v_{\min }\right) \leq I(v), \quad v_{\min }, v \in V
$$

To find the vector $v_{\min }$ numerical methods can be applied. An example will be shown in the next section.

\section{Example}

As an example consider an experimental heat plant, shown in Figure 1. It has the form of a thin copper rod $30 \mathrm{~cm}$ long with an electric heater of the length $\Delta x_{u}$ localized at one end and resistive temperature sensor long $\Delta x$ at the other end. The input signal of the system is the standard current signal $0-5[\mathrm{~mA}]$. It is amplified to the range $0-1.5[\mathrm{~A}]$ and it is the input signal for the heater. The temperature of the rod is measured with the use of a resistance sensor. The signal from the sensor is transformed to the standard current signal $0-5[\mathrm{~mA}]$ with the use of a transducer. The plant can be described with the use of different mathematical models.

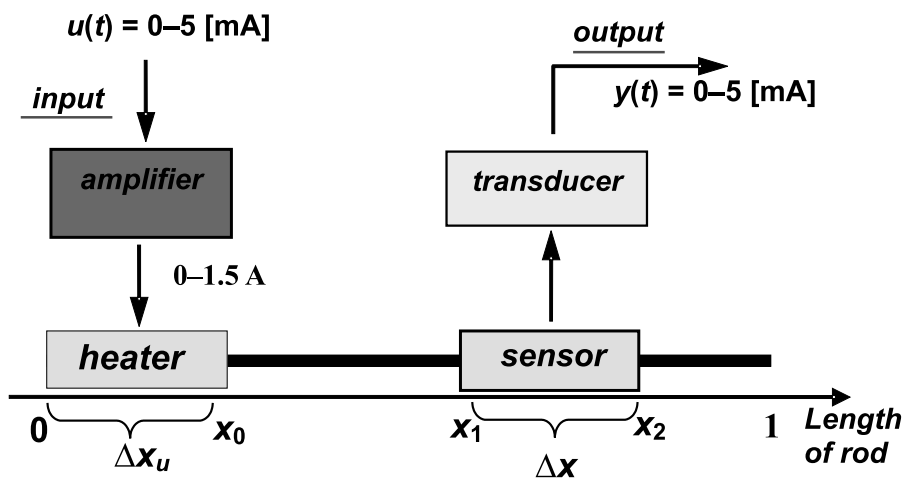

Fig. 1. The experimental heat control plant 
The step response of the plant is presented in Figure 2.

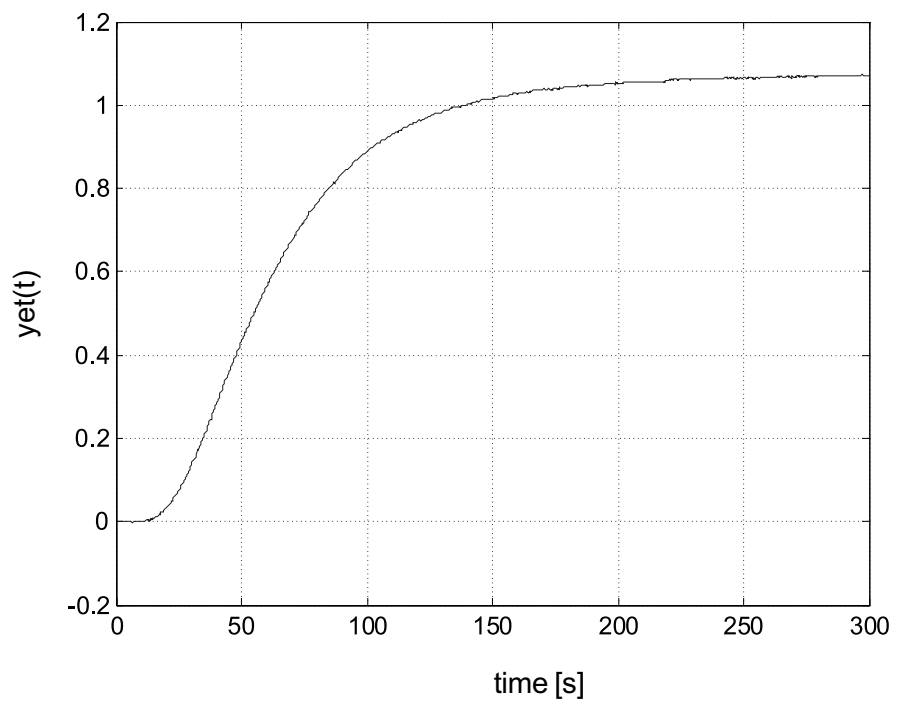

Fig. 2. The step response of the experimental plant

The mathematical model of the control plant we deal with is the following heat transfer equation:

$$
\left\{\begin{array}{l}
\frac{\partial T(x, t)}{\partial t}=a \frac{\partial^{2} T(x, t)}{\partial x^{2}}-R_{a} T(x, t)+b(x) u(t), \quad 0 \leq x \leq 1, \quad t \geq 0 \\
\frac{\partial T(0, t)}{\partial x}=0, \quad \frac{\partial T(1, t)}{\partial x}=0, \quad t \geq 0 \\
T(x, 0)=0, \quad 0 \leq x \leq 1 \\
y(t)=y_{0}^{1} T(x, t) c(x) d x
\end{array}\right.
$$

where $\theta(x, t)$ denotes the temperature of the rod at the point $x \in[0,1]$ and time moment $t, a$ and $R_{a}$ are the suitable heat transfer coefficients. All variables and constants are dimensionless. The coefficient $R_{a}$ describes the heat exchange along the side surface of the rod. The coefficient $y_{0}$ is the gain of the slotted line. The heat exchange at the ends of rod is much smaller than along the side surface and it can be described by the homogeneous Neumann's boundary conditions. To make the model simpler, the length of the rod was assumed equal to one: $x \in[0,1]$. The diameter of the rod is much smaller than its length, that is why in equation (10) 
only length of rod $x$ is considered. The control and observation in the plant we deal with (see Fig. 1) are distributed. Both the heater characteristic function $b(x)$ and the sensor characteristic function $c(x)$ depend on the length of these elements. The length of the heater is equal to $\Delta x_{u}=1 / 13$ and its characteristic function is as follows:

$$
b(x)=\left\{\begin{array}{lll}
1 & \text { for } & x \in\left(0, x_{0}\right) \\
0 & \text { for } & x \notin\left(0, x_{0}\right)
\end{array}\right.
$$

where $x_{0}=1 / 13$. The temperature of the rod is measured in the segment $\Delta x=x_{2}-x_{1}=1 / 13$ (distributed observation). The temperature sensor is described by the following characteristic function:

$$
c(x)=\left\{\begin{array}{lll}
1 & \text { for } & x \in\left(x_{1}, x_{2}\right) \\
0 & \text { for } & x \notin\left(x_{1}, x_{2}\right)
\end{array}\right.
$$

where $x_{1}=25 / 52, x_{2}=27 / 52$.

The numerical values of heat exchange coefficients $a$ and $R_{a}$ were calculated with the use of the least squares method, the experimental step response shown in Figure 2 and the cost function (8). They are equal to $a=0.000945, R_{a}=0.0271$.

The value of the steady-state gain $y_{0}$ was calculated via comparison of $y(k h)$ with $\tilde{y}(k h)$ after a suitably long time $t_{S}=S h$. The value of this gain is equal to 25.7922 . Let $L(U, X)$ denote the space of linear continuous operators $S: U \rightarrow X$ with the following natural norm: $\|S\|=\sup \left\{\|S v\|_{X}:\|v\|_{U} \leq 1\right\}$. Let $X=L^{2}(0,1 ; \boldsymbol{R})$ be a Hilbert space with scalar product $(p \mid d)=\int_{0}^{1} p(x) d(x) d x$. The boundary problem (10) can be interpreted (Sakawa [16], Pazy [13, p. 106], Kato [5, p. 488]) as the following differential equation in the Hilbert space $L^{2}(0,1 ; \boldsymbol{R})$ :

$$
\left\{\begin{array}{l}
\dot{\theta}(t)=A \theta(t)+B u(t), \quad \theta(0)=0, \quad 0 \leq t \\
y(t)=C T(t)
\end{array}\right.
$$

where $\theta(t) \in X=L^{2}(0,1 ; \boldsymbol{R}), u(t) \in \boldsymbol{R}=U, y(t) \in \boldsymbol{R}=Y$, $B \in L(U, X),(B u(t))(x)=b(x) u(t), C \in L(X, Y), C T(t)=y_{0}(T(t) \mid c), A$ is a linear operator with the domain $D(A)$ :

$$
\begin{aligned}
& A w=a w^{\prime \prime}-R_{a} w, \quad w^{\prime \prime}(x)=d^{2} w(x) / d x^{2}, \\
& D(A)=\left\{w \in X: w^{\prime \prime} \in X, w^{\prime}(0)=0, w^{\prime}(1)=0\right\}
\end{aligned}
$$


The operator defined by (14) is self-adjoint and has a compact resolvent. This implies that $A$ is a discrete operator. Spectrum of discrete operator consists only of isolated eigenvalues and all eigenvalues have finite multiplicities. The operator given by (14) has a discrete spectrum with the simple eigenvalues:

$$
\lambda_{i}=-i^{2} a \pi^{2}-R_{a}, \quad i=0,1,2,3, \ldots
$$

Eigenvectors associated with the eigenvalues (15) are defined as follows:

$$
w_{i}(x)=\left\{\begin{array}{l}
1 \quad, \quad i=0 \\
\sqrt{2} \cos (i \pi x), i=1,2, \ldots
\end{array}\right.
$$

The eigenvectors (16) build an orthonormal basis of the space $X=L^{2}(0,1 ; \boldsymbol{R})$. If the basis of $X=L^{2}(0,1 ; \boldsymbol{R})$ is built by the set of eigenvectors (16), then the operators $A, B$ and $C$ in (4) can be expressed as the following infinite-dimensional matrices (see Triggiani (1975) [17]):

$$
A=\operatorname{diag}\left\{\lambda_{0}, \lambda_{1}, \lambda_{2}, \ldots\right\}, \quad B=\left[b_{0} b_{1} b_{2} \ldots\right]^{T}, \quad C=y_{0}\left[c_{0} c_{1} c_{2} \ldots\right]
$$

where $b_{i}=\int_{0}^{1} b(x) w_{i}(x) d x=\left(b \mid w_{i}\right), c_{i}=\int_{0}^{1} c(x) w_{i}(x) d x=\left(c \mid w_{i}\right), i=0,1,2, \ldots$

The operator (5) is the generator of an analytical, exponentially stable $C_{0}$-semigroup $e^{A t}, t \geq 0$ in the space $X=L^{2}(0,1 ; \boldsymbol{R})$, where:

$$
e^{A t} w=\sum_{i=0}^{\infty} e^{\lambda_{i} t}\left(w \mid w_{i}\right) w_{i}
$$

The analysis of (15) and (18) justifies the use of finite-dimensional approximation of (13). Instead of $i=0,1,2,3, \ldots$ we will use: $i=0,1,2,3, \ldots, N$. A suitable value of $N$ for finite-dimensional approximation is $N=25$ (see Oprzędkiewicz (1995) [10]). The respective finite-dimensional matrix representations of the operators $A, B$ and $C$ for the presented model (13) are shown in Table 1.

Alternative models of this plant have the form of transfer functions with delay or without delay (see for example Oprzędkiewicz (2001) [12]). The Kupfmuller's model is expressed as underneath:

$$
G_{k}(s)=\frac{k e^{-\tau s}}{\left(T_{1} s+1\right)\left(T_{2} s+1\right)}
$$


Parameters of model (19) were calculated with the use of the least square method and they are given also in Table 1. To modeling the delay in MATLAB the Padee approximation was applied.

Furthermore, the Strejc's model without delay is described as follows:

$$
G_{k}(s)=\frac{k}{\left(T_{n} s+1\right)^{n}}
$$

Parameters of the model (20) were calculated with the use of the least square method and they are given also in Table 1.

Table 1

Parameters of alternative models of system under consideration

\begin{tabular}{|c|c|}
\hline Model & Parameters \\
\hline \multirow{3}{*}{$\begin{array}{l}\text { State-space model described } \\
\text { by (13)-(17) }\end{array}$} & $\begin{array}{l}A=\operatorname{diag}\{-0.0271,-0.0364,-0.0644,-0.1110,-0.1763,-0.2603, \\
-0.3629,-0.4841,-0.6240,-0.7826,-0.9598,-1.1556,-1.3702, \\
-1.6033,-1.8551,-2.1256,-2.4148,-2.7225,-3.0490,-3.3941, \\
-3.7578,-4.1402,-4.5413,-4.9610,-5.3993\},\end{array}$ \\
\hline & 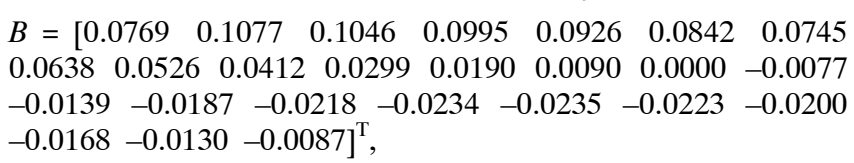 \\
\hline & $\begin{array}{l}C=\left[\begin{array}{llllllll}0.9920 & 0.0000 & -1.3995 & 0.0000 & 1.3893 & 0.0000 & -1.3724 \\
0.0000 & 1.3489 & 0.0000 & -1.3191 & 0.0000 & 1.2832 & 0.0000 & -1.2415 \\
0.0000 & 1.1944 & 0.0000 & -1.1423 & 0.0000 & 1.0856 & 0.0000 & -1.0248 \\
0.0000 & 0.9605\end{array}\right]\end{array}$ \\
\hline $\begin{array}{l}\text { Kupfmuller's model } \\
\text { described by (19) }\end{array}$ & $\begin{array}{l}T_{1}=38.1392 \\
T_{2}=14.8068 \\
\tau=15.0153 \\
\text { Order of Pade approximation: } 10\end{array}$ \\
\hline $\begin{array}{l}\text { Strejc's model described } \\
\text { by }(20)\end{array}$ & $\begin{array}{l}T_{n}=16.3623 \\
n=3\end{array}$ \\
\hline
\end{tabular}

For the above plant a number of simulations was run. The minimum of cost function (8) was found with the use of MATLAB function fminsearch, the starting parameters for optimization were equal: $\alpha=0.5, T_{m}=22[\mathrm{~s}], T_{\alpha}=15[\mathrm{~s}] \omega_{l}=0.001, \omega_{h}=0.5$. Results of are shown in Table 1. The best model is described by row in table and shown in Figure 3.

To compare the considered SSF model with the previously built, most accurate models of the system we deal with in Table 3 values of cost functions for all previous models are remembered. 
Table 2

Results of simulations and parameters of SSF model

\begin{tabular}{|c|c|c|c|c|c|c|c|}
\hline $\begin{array}{c}\text { Order } \\
\text { of Strejc } \\
\text { model } M\end{array}$ & $\begin{array}{c}\text { Order of } \\
\text { ORA appro- } \\
\text { ximation } N\end{array}$ & $\begin{array}{c}\text { Fractional } \\
\text { order } \\
\alpha\end{array}$ & $\begin{array}{c}\text { Time } \\
\text { constant } \\
T_{M}[\mathrm{~s}]\end{array}$ & $\begin{array}{c}\text { Time } \\
\text { constant } \\
T_{\alpha}[\mathrm{s}]\end{array}$ & $\begin{array}{c}\text { Low } \\
\text { frequency } \\
\omega_{l}\end{array}$ & $\begin{array}{c}\text { High } \\
\text { frequency } \\
\omega_{h}\end{array}$ & $\begin{array}{c}\text { Cost } \\
\text { function } \\
(8)\end{array}$ \\
\hline 3 & 2 & 0.0727 & 21.5102 & 9.2338 & 0.0000 & 1.1456 & 0.0417 \\
\hline 3 & 3 & 0.5060 & 19.2617 & 0.8708 & 0.0031 & 1.0225 & 0.0341 \\
\hline $\mathbf{3}$ & $\mathbf{5}$ & $\mathbf{0 . 9 2 6 4}$ & $\mathbf{1 2 . 6 7 2 5}$ & $\mathbf{2 0 9 . 2 3 1 8}$ & $\mathbf{0 . 0 0 0 0}$ & $\mathbf{1 5 . 1 2 6 0}$ & $\mathbf{0 . 0 0 7 9}$ \\
\hline 3 & 10 & 0.8722 & 13.0862 & 65.2818 & 0.0038 & 1.04 .4430 & 0.0106 \\
\hline 2 & 2 & 3.6548 & 32.4798 & 12.4378 & 0.0011 & 0.0082 & 0.0152 \\
\hline 2 & 3 & 1.3459 & 31.3981 & 53.0259 & 0.0000 & 0.4961 & 0.0174 \\
\hline 2 & 5 & 0.9759 & 21.4935 & 87.5136 & 0.0003 & 4.4562 & 0.0535 \\
\hline 2 & 10 & -1.0312 & 47.5752 & 69.4634 & 0.0146 & 0.0250 & 0.2608 \\
\hline
\end{tabular}

Table 3

Comparison of the SSF model to other models of considered experimental plant

\begin{tabular}{|l|c|c|}
\hline \multicolumn{1}{|c|}{ Model } & The summarized order of model & Value of cost function (8) \\
\hline State-space & 25 & 0.0156 \\
\hline 2'nd order Kupfmuller model & 12 & 0.0039 \\
\hline Strejc model without delay & 3 & 0.1514 \\
\hline SSF model & 8 & 0.0079 \\
\hline
\end{tabular}

The step responses of plant and the SSF model are shown in Figure 3.

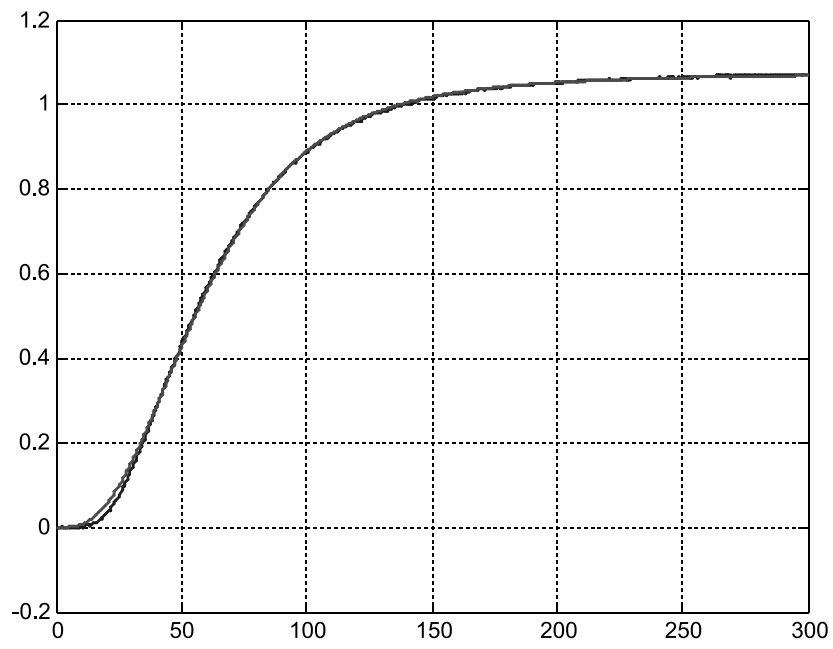

Fig. 3. The step responses of plant and the SSF model 


\section{Conclusions}

Final conclusions form paper can be formulated as follows:

- The proposed SSF model allows us to precisely model an infinite-dimensional plant and order of this model is lower, than order of other models with comparable accuracy.

- The model can be easily implemented in MATLAB/SIMULINK platform and their parameters can be calculated with the use of standard optimization tools

- The further investigations of the considered model cover a broad area of problems, for example analytical estimations of model parameters.

\section{References}

[1] Busłowicz M., Stability of linear continuous time fractional order systems with delay of the retarder type. Bull. Pol. Acad. Sci. Tech., vol. 56, No. 4, 2008, 319-324.

[2] Chen Y.Q., Oustaloup-Recursive-Approximation for Fractional Order Differentiators. MathWorks, Inc. Matlab Central File Exchange, 2003, URL: www.mathworks.com/matlabcentral/ fileexchange $/ 3802$

[3] Das S., Functional fractional calculus for system identification and controls. Springer, Berlin, 2008.

[4] Kaczorek T., Selected Problems of Fractional Systems Theory. Springer, Berlin, 2011.

[5] Kato T. (): Perturbation theory for linear operator. Springer, Berlin, 1980.

[6] De Larminat P. (Ed.), Analysis and Control of Linear Systems. Wiley and sons, ISTE 2007.

[7] Merrikh-Bayat F., Rules for selecting the parameters of Oustaloup recursive approximation for the simulation of linear feedback systems containing $P I^{\alpha} D^{\mu}$ controller. Commun Nonlinear Sci Numer Simulat, 17, 2012, 1852-1861.

[8] Mitkowski W. Oprzędkiewicz K., A sample time optimization problem in a digital control system. System modeling and optimization: 23rd IFIP TC 7 conference: Cracow, Poland, July 23-27, 2007: revised selected papers, eds. Adam Korytowski [et al.] Berlin; Heidelberg; New York, 2009, 382-396.

[9] Mitkowski W., Obrączka A., Simple identification of fractional differential equation. Solid State Phenomena, vol. 180, 2012, 331-338.

[10] Oprzędkiewicz K., Modeling of parabolic problems in MATLAB/SIMULINK. Proc. of III Conf. Comp. Simulation, Nov. 15-17, Mexico City, 1995, 45-49.

[11] Oprzędkiewicz K., An example of parabolic system identification. Zeszyty Naukowe AGH, Elektrotechnika, t. 16, z. 2, 1997, 99-106 (in Polish).

[12] Oprzędkiewicz K., The discrete cancellation controller and his practical implementation. Pomiary, Automatyka, Robotyka (PAR), r. 5, nr 11, 2001, 8-15 (in Polish).

[13] Pazy A., Semigroups of Linear Operators and Applications to Partial Differential Equations. Springer, New York, 1983.

[14] Podlubny I., Fractional differential equations. Academic Press, San Diego, 1999.

[15] Podlubny I., Fractional-order systems and $P I^{\alpha} D^{\mu}$-controllers". IEEE Trans Autom Control, 44(1), 1999, 208-214.

[16] Sakawa Y., Feedback stabilization of linear diffusion systems. SIAM J, Control and Optimization, vol. 21, No. 5, 1983, 667-676.

[17] Triggiani R., On the stabilization problem in Banach space. J. Math. Anal. Appl., vol. 52, No. 3, 1975, 383-403. 